\title{
FOAM Regimen
}

National Cancer Institute

\section{Source}

National Cancer Institute. FOAM Regimen. NCI Thesaurus. Code C10279.

A chemotherapy regimen consisting of fluorouracil, vincristine, doxorubicin, and mitomycin that may be used for the treatment of breast cancer. 